Pacific

Journal of

Mathematics

ON THE ASYMPTOTICS OF THE TRACE OF THE HEAT KERNEL FOR THE MAGNETIC SCHRÖDINGER

OPERATOR

Junichi ARAMAKI

Volume 198 No. 1

March 2001 


\title{
ON THE ASYMPTOTICS OF THE TRACE OF THE HEAT KERNEL FOR THE MAGNETIC SCHRÖDINGER OPERATOR
}

\author{
Junichi ARAMAKI
}

\begin{abstract}
We consider the effect of a magnetic field for the asymptotic behavior of the trace of the heat kernel for the Schrödinger operator. We discuss the case where the operator has compact resolvents in spite of the fact that the electric potential is degenerate on some submanifold. According to the degree of the degenaracy, we obtain the classical and non-classical asymptotics.
\end{abstract}

\section{Introduction.}

In this paper, we consider the Schrödinger operator on $\mathbb{R}^{d}$ with the magnetic vector potential $A(z)$ and the electric scalar potential $V(z)$ :

$$
H(A, V)=\frac{1}{2}(i \nabla+A(z))^{2}+V(z) .
$$

We will assume that $H(A, V)$ and $H(0, V)$ are essentially self-adjoint in $L^{2}\left(\mathbb{R}^{d}\right)$ starting from $C_{0}^{\infty}\left(\mathbb{R}^{d}\right)$ and we denote the self-adjoint extensions by $H$ and $H_{0}$, respectively. It is well known that if

$$
\lim _{|z| \rightarrow \infty} V(z)=+\infty
$$

$H_{0}$ has compact resolvents (c.f. for example, Reed and Simon [8]). Odencrantz [7] studied the asymptotic behavior of $\operatorname{Tr}\left[\exp (-t H)-\exp \left(-t H_{0}\right)\right]$ as $t \downarrow 0$ in the case where $V(z) \approx|z|^{2 p}(p>0)$ and the curl of $A(z)$ is a uniform magnetic field. Matsumoto [5] extended the result to the case with more general magnetic field.

However, (1.2) is not a necessary condition in order that $H_{0}$ has compact resolvents. In spite of the lack of (1.2), there are some cases where $H_{0}$ has compact resolvents.

The motivation of this paper originates in the works of Simon [11], Robert [9] and Aramaki [3]. In order to explain this, we put

$$
Z_{\mathrm{cl}}(t)=(2 \pi)^{-d} \iint e^{-t\left(|\zeta|^{2} / 2+V(z)\right)} d z d \zeta .
$$

It is well known that $\operatorname{Tr}\left[\exp \left(-t H_{0}\right)\right] \leq Z_{\mathrm{cl}}(t)$ for $t>0$. In [11], he considered the degenerate potential $V(z)$ of the form $V(x, y)=|x|^{2 p}|y|^{2 q}(p, q>0)$ on 
$\mathbb{R}^{2}$. Then it holds that $H_{0}$ has compact resolvents. In this case, $Z_{\mathrm{cl}}(t) \equiv \infty$ while $\operatorname{Tr}\left[\exp \left(-t H_{0}\right)\right]<\infty$ for all $t>0$. He succeeded to get the asymptotics of $\operatorname{Tr}\left[\exp \left(-t H_{0}\right)\right]$ as $t \downarrow 0$ by using the sliced Golden-Thompson inequality and the sliced bread inequality according to the cases $p \neq q$ and $p=q$. $[\mathbf{9}]$ and $[\mathbf{3}]$ considered a slightly modified potential $V(z)$ of the form

$$
V(z)=\left(1+|x|^{2}\right)^{p}|y|^{2 q} \quad(p, q>0 \text { integers }), z=(x, y) \in \mathbb{R}^{d}=\mathbb{R}^{n} \times \mathbb{R}^{m} .
$$

Then $H_{0}$ also has compact resolvents (cf. [9]). In this case, it is easy to see that $Z_{\mathrm{cl}}(t)$ is finite for $t>0$ in the case $p m>q n$, however, $Z_{\mathrm{cl}}(t)$ is infinite for $t>0$ in the case $p m \leq q n$. In the present paper, we consider the magnetic Schrödinger operator $H$ with an electric potential $V(z)$ of the type (1.3). We want to show that if the magnetic potential $A(z)$ is relatively benign, the difference between $\operatorname{Tr}[\exp (-t H)]$ and $\operatorname{Tr}\left[\exp \left(-t H_{0}\right)\right]$ can be controlled by using the representation of the heat kernels in terms of Wiener integrals. From this, we can see the asymptotics of $\operatorname{Tr}[\exp (-t H)]$ as $t \downarrow 0$, if we combined the estimate with our previous results as follows.

We denote the numbers of the eigenvalues counting multiplicities of $H$ and $H_{0}$ equal to or less than $\lambda$ by $N(\lambda)$ and $N_{0}(\lambda)$, respectively. Then Aramaki [3] obtained the following.

Proposition 1.1. Under (1.3), there exists $\delta>0$ such that:

(i) If $p m>q n, N_{0}(\lambda)=c_{1} \lambda^{(m+m q+n q) /(2 q)}\left(1+O\left(\lambda^{-\delta}\right)\right)$ as $\lambda \rightarrow \infty$.

(ii) If $p m=q n, N_{0}(\lambda)=c_{2} \lambda^{(m+m q+n q) /(2 q)} \log \lambda+c_{3} \lambda^{(m+m q+n q) /(2 q)}(1+$ $\left.O\left(\lambda^{-\delta}\right)\right)$ as $\lambda \rightarrow \infty$.

(iii) If $p m<q n, N_{0}(\lambda)=c_{4} \lambda^{n(1+p+q) /(2 p)}\left(1+O\left(\lambda^{-\delta}\right)\right)$ as $\lambda \rightarrow \infty$ where $c_{i}(i=1,2,3,4)$ are some positive constants which can be calculated concretely.

Here we note that in only the case where $p m>q n$, the problem is classical in the sense of

$$
\operatorname{vol}\left\{(z, \zeta) \in \mathbb{R}^{d} \times \mathbb{R}^{d} ; \frac{1}{2}|\zeta|^{2}+V(z) \leq \lambda\right\}<\infty .
$$

On the other hand, in the case where $p m \leq q n$, the problem is non-classical in the sense that the left hand side of (1.4) is the infinity. It easily follows from the well known equation

$$
\operatorname{Tr}\left[\exp \left(-t H_{0}\right)\right]=\int \exp (-t \lambda) d N_{0}(\lambda)
$$

that we also have the asymptotic behavior of $\operatorname{Tr}\left[\exp \left(-t H_{0}\right)\right]$ as $t \downarrow 0$.

Proposition 1.2. Under (1.3), we have: 
(i) If $p m>q n, \operatorname{Tr}\left[e^{-t H_{0}}\right]=d_{1} t^{-(m+m q+n q) /(2 q)}\left(1+O\left(t^{\delta}\right)\right)$ as $t \rightarrow 0$.

(ii) If $p m=q n$,

$\operatorname{Tr}\left[e^{-t H_{0}}\right]=d_{2} t^{-(m+m q+n q) /(2 q)} \log t^{-1}+d_{3} t^{-(m+m q+n q) /(2 q)}\left(1+O\left(t^{\delta}\right)\right)$ as $t \rightarrow 0$.

(iii) If $p m<q n, \operatorname{Tr}\left[e^{-t H_{0}}\right]=d_{4} t^{-n(1+p+q) /(2 p)}\left(1+O\left(t^{\delta}\right)\right)$ as $t \rightarrow 0$

where $d_{i}(i=1,2,3,4)$ are positive constants which can easily be given by $c_{i}$ (also given in Section 4) and $\delta$ is as in Proposition 1.1.

For the precise, see Aramaki [1], [2], [3], [9] and [11].

In this paper, we shall treat the case where $V(z)$ is of the type of (1.3). Under some hypotheses on the magnetic vector potential $A(z)$, we shall obtain the asymptotic behavior of $\operatorname{Tr}\left[\exp (-t H)-\exp \left(-t H_{0}\right)\right]$ as $t \downarrow 0$ is better than that in Proposition 1.2 in each case. Therefore, we see that the magnetic field does not give any influence to the leading terms of the asymptotics. One of the features of this paper is that the argument is based on the probabilistic representations of the heat kernels. As we do not use any pseudodifferential calculus in the proof of the main theorem, it suffices to assume less smoothness of $V$ and $A$.

The plan of this paper is as follows. In $\S 2$, we give the main theorem. Section 3 is devoted to the proof of the main theorem. In $\S 4$, we give an example so that the electric potential $V(x, y)$ is of the form (1.3).

\section{Hypotheses and Statements.}

Let $\mathbb{R}^{d}=\mathbb{R}^{n} \times \mathbb{R}^{m}$ and we write a variable $\mathrm{z}$ in $\mathbb{R}^{d}$ by $z=(x, y) \in \mathbb{R}_{x}^{n} \times \mathbb{R}_{y}^{m}$. We consider the operator:

$$
H(A, V)=\frac{1}{2}\left(i \nabla_{(x, y)}+A(x, y)\right)^{2}+V(x, y)
$$

where $i=\sqrt{-1}$ and $\nabla_{(x, y)}$ denotes the gradient operator. First of all, we state the assumptions on the scalar potential $V(x, y)$.

(V.1) $V(x, y) \in C^{1}\left(\mathbb{R}^{n} \times \mathbb{R}^{m}\right)$ is a real valued function.

(V.2) There exist positive constants $p, q$ and $C>0$ such that

$$
V(x, y) \geq C\left(1+|x|^{2}\right)^{p}|y|^{2 q} \text { for all }(x, y) \in \mathbb{R}^{n} \times \mathbb{R}^{m} .
$$

Moreover, we give the assumptions for the vector potential $A(x, y)$ :

(A.1) $A(x, y)=\left(a_{1}(x, y), \ldots, a_{d}(x, y)\right) \in C^{2}\left(\mathbb{R}^{d} ; \mathbb{R}^{d}\right)$.

(A.2) There exist constants $a, b$ satisfying $0 \leq a<p, 0 \leq b<q,(q+1) a<$ $p(b+1)$ and $C_{1}>0$ such that for every $j=1,2, \ldots d$ and $|\alpha| \leq 2$,

$$
\left|\partial_{x, y}^{\alpha} a_{j}(x, y)\right| \leq C_{1}\left(1+|x|^{2}\right)^{a}|y|^{2 b} .
$$

By the assumptions (V.1) and (A.1), $H(A, V)$ is essentially self-adjoint in $L^{2}\left(\mathbb{R}^{d}\right)$ starting from $C_{0}^{\infty}\left(\mathbb{R}^{d}\right)$ (c.f. Schechter [10]) and we denote the unique self-adjoint extensions of $H(A, V)$ and $H(0, V)$ by $H$ and $H_{0}$, respectively 
as in introduction. Under (V.1), (V.2), $H_{0}$ has compact resolvents and $\exp \left(-t H_{0}\right)$ is of trace class, i.e., $\operatorname{Tr}\left[\exp \left(-t H_{0}\right)\right]$ is finite (c.f. [1]). Since $V$ is bounded from below, we have the diamagnetic inequality:

$$
e^{-t H(A, V)} \preceq e^{-t H(0, V)} \quad \text { for every } t>0,
$$

that is to say, for all $u \in L^{2}\left(\mathbb{R}^{d}\right)$,

$$
\left|\left(e^{-t H(A, V)} u\right)(z)\right| \leq\left(e^{-t H(0, V)}|u|\right)(z) \quad \text { a.e. } z \text { for } t>0 .
$$

Then it follows from Simon [13, p. 164] that

$$
\operatorname{Tr}\left[e^{-t H}\right] \leq \operatorname{Tr}\left[e^{-t H_{0}}\right] \text { for } t>0,
$$

so we see that $\exp (-t H)$ is also of trace class.

Then we have the main theorem.

Theorem 2.1. Under the conditions (V.1), (V.2), (A.1) and (A.2), we have the following.

(i) The case where $p m>q n$. If $q(4 a+n)-p(4 b+m) \neq 0$, we have

$$
\operatorname{Tr}\left[e^{-t H}-e^{-t H_{0}}\right]=O\left(t^{-(m+m q+n q) /(2 q)+\gamma_{1}}\right) \quad \text { as } t \downarrow 0
$$

and if $q(4 a+n)-p(4 b+m)=0$, we have

$$
\operatorname{Tr}\left[e^{-t H}-e^{-t H_{0}}\right]=O\left(t^{-(m+m q+n q) /(2 q)+\gamma_{1}} \log t^{-1}\right) \quad \text { as } t \downarrow 0
$$

where

$$
\gamma_{1}=\min \left\{\frac{2(q-b)}{q}, \frac{(1+q)(p m-q n)}{2 p q}+\frac{2(p(1+b)-a(q+1))}{p}\right\} .
$$

(ii) The case where $p m \leq q n$. If $q(4 a+n)-p(4 b+m) \neq 0$, we have

$$
\operatorname{Tr}\left[e^{-t H}-e^{-t H_{0}}\right]=O\left(t^{-n(1+p+q) /(2 p)+\gamma_{2}}\right) \quad \text { as } t \downarrow 0
$$

and if $q(4 a+n)-p(4 b+m)=0$, we have

$$
\operatorname{Tr}\left[e^{-t H}-e^{-t H_{0}}\right]=O\left(t^{-n(1+p+q) /(2 p)+\gamma_{2}} \log t^{-1}\right) \quad \text { as } t \downarrow 0
$$

where

$$
\gamma_{2}=\min \left\{\frac{2(q-b)}{q}+\frac{(1+q)(q n-p m)}{2 p q}, \frac{2(p(1+b)-a(q+1))}{p}\right\} .
$$

Remark 2.2. Since $\gamma_{1}$ and $\gamma_{2}$ are positive numbers in any cases according to (A.2), the hypothesis (A.2) on the magnetic potential certainly gives an effect to the asymptotics in each case.

Using the Karamata Tauberian theorem and [3], we have also asymptotics of distrubution function $N(\lambda)$ of eigenvalues of $H$. 
Corollary 2.3. Addition to the hypotheses of Theorem 2.1, we assume that there exists $C_{2}>0$ such that

$$
V(x, y) \leq C_{2}\left(1+|x|^{2}\right)^{p}|y|^{2 q} .
$$

Then we have

$$
N(\lambda)=N_{0}(\lambda)(1+o(1)) \quad \text { as } \lambda \rightarrow \infty .
$$

Here $N_{0}(\lambda)$ is of the form:

(i) If $p m>q n$, we have

$$
N_{0}(\lambda)=c_{1} \lambda^{(m+m q+n q) /(2 q)}(1+o(1)) \quad \text { as } \lambda \rightarrow \infty .
$$

(ii) If $p m=q n$, we have

$$
N_{0}(\lambda)=c_{2} \lambda^{(m+m q+n q) /(2 q)} \log \lambda(1+o(1)) \quad \text { as } \lambda \rightarrow \infty .
$$

(iii) If $p m<q n$, we have

$$
N_{0}(\lambda)=c_{4} \lambda^{n(1+q+p) /(2 p)}(1+o(1)) \quad \text { as } \lambda \rightarrow \infty .
$$

Here $c_{1}, c_{2}, c_{4}$ are positive constants as in Proposition 1.1. For the precise values of the constants, see [3].

\section{Proof of the main theorem.}

In this section, we give the proof of Theorem 2.1. Let $p_{0}\left(t ; z, z^{\prime}\right)$ and $p\left(t ; z, z^{\prime}\right)$ be the distribution kernels of $\exp \left(-t H_{0}\right)$ and $\exp (-t H)$, respectively. Then, by the Feynman-Kac-Itô formula, we can write these heat kernels using probabilistic representations as follows.

$$
\begin{aligned}
p_{0}\left(t ; z, z^{\prime}\right)= & (2 \pi t)^{-d / 2} e^{-\left|z-z^{\prime}\right|^{2} /(2 t)} E_{0,0}^{0,0} \\
& \cdot\left[\exp \left(-t \int_{0}^{1} V\left(z+s\left(z-z^{\prime}\right)+\sqrt{t} Z_{s}\right) d s\right)\right], \\
p\left(t ; z, z^{\prime}\right)= & (2 \pi t)^{-d / 2} e^{-\left|z-z^{\prime}\right|^{2} /(2 t)} E_{0,0}^{0,0} \\
& \cdot\left[\exp \left(i F^{t}\left(z, z^{\prime}\right)-t \int_{0}^{1} V\left(z+s\left(z-z^{\prime}\right)+\sqrt{t} Z_{s}\right) d s\right)\right]
\end{aligned}
$$

where $F^{t}\left(z, z^{\prime}\right)=\sqrt{t} \int_{0}^{1} A\left(z+s\left(z-z^{\prime}\right)+\sqrt{t} Z_{s}\right) \circ d Z_{s}$. Here $E_{0,0}^{0,0}$ is the expectation with respect to the $d(=n+m)$ - dimensional pinned Brownian motion $\left\{Z_{s}\right\}_{0 \leq s \leq 1}=\left\{X_{s}, Y_{s}\right\}_{0 \leq s \leq 1}=\left\{X_{s}^{1}, \ldots, X_{s}^{n}, Y_{s}^{1}, \ldots, Y_{s}^{m}\right\}_{0 \leq s \leq 1}$ such that $Z_{0}=0=(0,0)$ and $Z_{1}=0=(0,0)$ and $\circ d Z_{s}$ denotes the Stratonovich integral. For the theory of these probabilistic facts, see $[\mathbf{1 3}]$ and [5]. Throughout this paper, we denote $E_{0,0}^{0,0}$ simply by $E$. We note that under (V.1) and (A.1), $p_{0}\left(t ; z, z^{\prime}\right)$ and $p\left(t ; z, z^{\prime}\right)$ are continuous with respect to $t>0$ and $z, z^{\prime} \in \mathbb{R}^{d}$. Since we study the traces of $\exp \left(-t H_{0}\right)$ and 
$\exp (-t H)$, it suffices to consider the heat kernels on the diagonal set only. I.e.,

$$
\begin{aligned}
p_{0}(t ; z, z) & =(2 \pi t)^{-d / 2} E\left[\exp \left(-t \int_{0}^{1} V\left(z+\sqrt{t} Z_{s}\right) d s\right)\right] \\
p(t ; z, z) & =(2 \pi t)^{-d / 2} E\left[\exp \left(i F^{t}(z)-t \int_{0}^{1} V\left(z+\sqrt{t} Z_{s}\right) d s\right)\right]
\end{aligned}
$$

where $F^{t}(z)=\sqrt{t} \int_{0}^{1} A\left(z+\sqrt{t} Z_{s}\right) \circ d Z_{s}$

Let $\left\{Z_{s}\right\}_{0 \leq s \leq 1}=\left\{X_{s}, Y_{s}\right\}_{0 \leq s \leq 1}$ be defined on a probability space $(\Omega, \mathcal{F}, P)$ and define

$$
\xi=\sup _{0 \leq s \leq 1}\left|X_{s}\right|
$$

Then it follows from Lévy's work that:

Lemma 3.1. For every $R>0$,

$$
P(\xi \geq R) \leq 2 n e^{-2 R^{2} / n}
$$

For the proof, see Simon [12], Itô and Mckean [4] and [5, Lemma 1].

From now, we denote various constants independent of $t>0$ and $(x, y) \in$ $\mathbb{R}^{n} \times \mathbb{R}^{m}$ by the same notations $C, C_{j}(j=1,2, \ldots)$ etc..

Lemma 3.2. Under the assumptions (A.1) and (A.2), there exists a constant $C>0$ such that

$$
E\left[\left|F^{t}(x, y)\right|^{4}\right] \leq C t^{4}\left(1+|x|^{2}\right)^{4 a}|y|^{8 b}
$$

for all $(x, y) \in \mathbb{R}^{n} \times \mathbb{R}^{m}$ and $t \in(0,1)$.

Proof. Since the proof is essentially the same as [5, Lemma 2], we give only an outline of the proof.

Let $\left\{w_{s}\right\}_{0 \leq s \leq 1}$ be the standard $d$-dimensional Brownian motion defined on a probability space $(\tilde{\Omega}, \tilde{\mathcal{F}}, \tilde{P})$. Then, the pinned Brownian motion $\left\{Z_{s}\right\}_{0 \leq s \leq 1}$ such that $Z_{0}=Z_{1}=0$ is the solution of the stochastic differential equation:

$$
d Z_{s}^{i}=d w_{s}^{i}-\frac{Z_{s}^{i}}{1-s} d s \quad(0<s<1), \quad Z_{0}^{i}=0 \quad(i=1,2, \ldots, d) .
$$


Thus, by the Itô formula, we have

$$
\begin{aligned}
F^{t}(z)= & t \sum_{i, j=1}^{d} \int_{0}^{1} d w_{s}^{i} \int_{0}^{s} \partial_{j} a_{i}\left(z+\sqrt{t} Z_{u}\right) d w_{u}^{j} \\
& -t \sum_{i, j=1}^{d} \int_{0}^{1} d w_{s}^{i} \int_{0}^{s} \partial_{j} a_{i}\left(z+\sqrt{t} Z_{u}\right) \frac{Z_{u}^{j}}{1-u} d u \\
& +\frac{1}{2} t^{3 / 2} \sum_{i, j=1}^{d} \int_{0}^{1} d w_{s}^{i} \int_{0}^{s} \partial_{j}^{2} a_{i}\left(z+\sqrt{t} Z_{u}\right) d u \\
& -t \sum_{i, j=1}^{d} \int_{0}^{1} \frac{Z_{s}^{i}}{1-s} d s \int_{0}^{s} \partial_{j} a_{i}\left(z+\sqrt{t} Z_{u}\right) d w_{u}^{j} \\
& +t \sum_{i, j=1}^{d} \int_{0}^{1} \frac{Z_{s}^{i}}{1-s} d s \int_{0}^{s} \partial_{j} a_{i}\left(z+\sqrt{t} Z_{u}\right) \frac{Z_{u}^{j}}{1-u} d u \\
& -\frac{1}{2} t^{3 / 2} \sum_{i, j=1}^{d} \int_{0}^{1} \frac{Z_{s}^{i}}{1-s} d s \int_{0}^{s} \partial_{j}^{2} a_{i}\left(z+\sqrt{t} Z_{u}\right) d u \\
& +\frac{1}{2} t \sum_{i=1}^{d} \int_{0}^{1} \partial_{i} a_{i}\left(z+\sqrt{t} Z_{u}\right) d s .
\end{aligned}
$$

Since $Z_{s}^{i}$ is the Gaussian random variable of mean 0 and variance $s(1-s)$, we have

$$
E\left[\left|Z_{s}^{i}\right|^{2 m}\right]=(2 m-1) ! !(s(1-s))^{m} \quad \text { for } \quad m=1,2, \ldots .
$$

Using this equality, the Hölder inequality and (A.2), we can prove the lemma.

Lemma 3.3. For every $x \in \mathbb{R}^{n}$, put $A_{|x|}=-\frac{1}{2} \Delta_{y}+c|x|^{2 p}|y|^{2 q}$ on $L^{2}\left(\mathbb{R}^{m}\right)$ where $c$ is a positive constant and let $e^{-t A_{|x|}}\left(y, y^{\prime}\right)$ be the kernel of $e^{-t A_{|x|}}$ and $J(t ;|x|, y)=e^{-t A_{|x|}}(y, y)$. Then, we have following:

(i) There exist constants $C_{j}(j=1,2,3)$ such that

$$
|J(t ; 1, y)| \leq C_{1} t^{-m / 2}\left(e^{-C_{2} t|y|^{2 q}}+e^{-C_{3}|y|^{2} / t}\right) .
$$

(ii) For every $\lambda>0$, $J(t ;|x|, y)=\lambda^{-m} J\left(\lambda^{-2} t ;\left|\lambda^{(1+q) / p} x\right|, \lambda^{-1} y\right) \quad$ for all $t>0,(x, y) \in \mathbb{R}^{n} \times \mathbb{R}^{m}$.

Proof. For (i), see Matsumoto [6, Lemma 3.1]. For (ii), it follows from the Feynman-Kac formula that

$$
J(t ;|x|, y)=(2 \pi t)^{-m / 2} E\left[e^{-t \int_{0}^{1} c|x|^{2 p}\left|y+\sqrt{t} Y_{s}\right|^{2 q} d s}\right] .
$$


Thus we have

$$
\begin{aligned}
J(t ;|x|, \lambda y) & =\lambda^{-m}\left(2 \pi \lambda^{-2} t\right)^{-m / 2} E\left[e^{-\lambda^{-2} t \int_{0}^{1} c\left|\lambda^{(1+q) / p} x\right|^{2 p}\left|y+\sqrt{\lambda^{-2}} Y_{s}\right|^{2 q} d s}\right] \\
& =\lambda^{-m} J\left(\lambda^{-2} t ;\left|\lambda^{(1+q) / p} x\right|, y\right) .
\end{aligned}
$$

Now, we give the proof of Theorem 2.1.

Proof of Theorem 2.1.

Since $p(t ; x, y, x, y)$ is a real valued function, using (3.1) and (3.2), we can write

$$
\begin{aligned}
I(t ; x, y) & \equiv\left|p(t ; x, y, x, y)-p_{0}(t ; x, y, x, y)\right| \\
& =(2 \pi t)^{-d / 2}\left|E\left[\left(\cos F^{t}(x, y)-1\right) e^{-t \int_{0}^{1} V\left(x+\sqrt{t} X_{s}, y+\sqrt{t} Y_{s}\right) d s}\right]\right| .
\end{aligned}
$$

Since $0 \leq 1-\cos \theta \leq \theta^{2} / 2$ for $\theta \in \mathbb{R}, I(t ; x, y)$ is estimated by

$$
\frac{1}{2}(2 \pi t)^{-d / 2} E\left[F^{t}(x, y)^{2} e^{-t \int_{0}^{1} V\left(x+\sqrt{t} X_{s}, y+\sqrt{t} Y_{s}\right) d s}\right] .
$$

By the Schwartz inequality, Lemma 3.2 and hypothesis (A.2) and (V.2), we have

$$
\begin{aligned}
I(t ; x, y) & \leq C_{1} t^{-d / 2} E\left[F^{t}(x, y)^{4}\right]^{1 / 2} E\left[e^{-2 t \int_{0}^{1} V\left(x+\sqrt{t} X_{s}, y+\sqrt{t} Y_{s}\right) d s}\right]^{1 / 2} \\
& \leq C_{2} t^{2-d / 2}\left(1+|x|^{2}\right)^{2 a}|y|^{4 b} E\left[e^{-C_{3} t \int_{0}^{1}\left(1+\left|x+\sqrt{t} X_{s}\right|^{2}\right)^{p}\left|y+\sqrt{t} Y_{s}\right|^{2 q} d s}\right]^{1 / 2} .
\end{aligned}
$$

Define the function $\xi$ by (3.3) and let $\chi$ be the characteristic function of the sets $\{\xi \geq|x| / 2 \sqrt{t}\}$.

Now we decompose

$$
K(t ; x, y) \equiv E\left[e^{-C_{3} t \int_{0}^{1}\left(1+\left|x+\sqrt{t} X_{s}\right|^{2}\right)^{p}\left|y+\sqrt{t} Y_{s}\right|^{2 q} d s}\right]
$$

into the form $K(t ; x, y)=\sum_{j=1}^{2} K_{j}(t ; x, y)$ where

$$
\begin{aligned}
& K_{1}(t ; x, y)=E\left[e^{-C_{3} t \int_{0}^{1}\left(1+\left|x+\sqrt{t} X_{s}\right|^{2}\right)^{p}\left|y+\sqrt{t} Y_{s}\right|^{2 q} d s} \chi\right] \\
& K_{2}(t ; x, y)=E\left[e^{-C_{3} t \int_{0}^{1}\left(1+\left|x+\sqrt{t} X_{s}\right|^{2}\right)^{p}\left|y+\sqrt{t} Y_{s}\right|^{2 q} d s}(1-\chi)\right] .
\end{aligned}
$$

Then we note that $K(t ; x, y)^{1 / 2} \leq \sum_{j=1}^{2} K_{j}(t ; x, y)^{1 / 2}$. At first, we consider $K_{1}(t ; x, y)$. Since $\left(1+\left|x+\sqrt{t} X_{s}\right|^{2}\right)^{p} \geq 1$, we see that

$$
\begin{aligned}
K_{1}(t ; x, y) & \leq E\left[e^{\left.-C_{3} t \int_{0}^{1}\left|y+\sqrt{t} Y_{s}\right|^{2 q}\right) d s}\right] E[\chi] \\
& =E\left[e^{\left.-C_{3} t \int_{0}^{1}\left|y+\sqrt{t} Y_{s}\right|^{2 q}\right) d s}\right] P(\{\xi \geq|x| / 2 \sqrt{t}\}) .
\end{aligned}
$$

By Lemma 3.1 and Lemma 3.3 (i), we have

$$
K_{1}(t ; x, y) \leq C_{4}\left(e^{-C_{5} t|y|^{2 q}}+e^{-C_{6}|y|^{2} / t}\right) e^{-C_{7}|x|^{2} / t} .
$$


Therefore,

$$
\begin{aligned}
K_{1}(t) & \equiv t^{2-d / 2} \iint\left(1+|x|^{2}\right)^{2 a}|y|^{4 b} K_{1}(t ; x, y)^{1 / 2} d x d y \\
& \leq C_{4} t^{2-d / 2} \int\left(1+|x|^{2}\right)^{2 a} e^{-C_{7}|x|^{2} / t} d x \int|y|^{4 b}\left(e^{-C_{5} t|y|^{2 q}}+e^{-C_{6}|y|^{2} / t}\right) d y .
\end{aligned}
$$

Since a simple computation leads to

$$
\int\left(1+|x|^{2}\right)^{2 a} e^{-C_{1}|x|^{2} / t} d x=O\left(t^{n / 2}\right) \quad \text { as } t \rightarrow 0
$$

and

$$
\int|y|^{4 b}\left(e^{-C_{5} t|y|^{2 q}}+e^{-C_{6}|y|^{2} / t}\right) d y=O\left(t^{-(4 b+m) /(2 q)}\right),
$$

we have an estimate of $K_{1}(t)$ :

$$
K_{1}(t) \leq C_{8} t^{2-m / 2-(4 b+m) /(2 q)}=O\left(t^{-(m+m q+n q) /(2 q)+2(1-b / q)+n / 2}\right) .
$$

Secondly, we consider $K_{2}(t ; x, y)$. Since we have $\left|x+\sqrt{t} X_{s}\right| \geq|x| / 2$ on $\operatorname{supp}(1-\chi)$,

$$
K_{2}(t ; x, y) \leq E\left[e^{\left.-C_{1} t \int_{0}^{1}\left(1+|x|^{2}\right)^{p}\left|y+\sqrt{t} Y_{s}\right|^{2 q}\right) d s}\right] .
$$

Now, we decompose

$$
\begin{aligned}
K_{2}(t) & \equiv t^{2-d / 2} \iint\left(1+|x|^{2}\right)^{2 a}|y|^{4 b} K_{2}(t ; x, y)^{1 / 2} d x d y \\
& =K_{2,1}(t)+K_{2,2}(t)
\end{aligned}
$$

where

$$
\begin{aligned}
& K_{2,1}(t)=t^{2-d / 2} \iint_{|x| \leq 1}\left(1+|x|^{2}\right)^{2 a}|y|^{4 b} K_{2}(t ; x, y)^{1 / 2} d x d y \\
& K_{2,2}(t)=t^{2-d / 2} \iint_{|x| \geq 1}\left(1+|x|^{2}\right)^{2 a}|y|^{4 b} K_{2}(t ; x, y)^{1 / 2} d x d y .
\end{aligned}
$$

For the estimate of $K_{2,1}(t)$, we use $\left(1+|x|^{2}\right)^{p} \geq 1$ in (3.9). Thus we have

$$
K_{2,1}(t) \leq t^{2-d / 2} \iint_{|x| \leq 1}\left(1+|x|^{2}\right)^{2 a}|y|^{4 b} E\left[e^{-C_{2} t \int_{0}^{1}\left|y+\sqrt{t} Y_{s}\right|^{2 q} d s}\right]^{1 / 2} d x d y .
$$

Here, by Lemma 3.3 (i),

$$
\begin{aligned}
E\left[e^{-C_{2} t \int_{0}^{1}\left|y+\sqrt{t} Y_{s}\right|^{2 q} d s}\right]^{1 / 2} & =\left\{(2 \pi t)^{m / 2} e^{-t\left(-\frac{1}{2} \Delta_{y}+C_{4}|y|^{2 q}\right)}(y, y)\right\}^{1 / 2} \\
& \leq C_{3} t^{m / 4}\left\{e^{-C_{4} t|y|^{2 q}}+e^{-C_{5}|y|^{2} / t}\right\} .
\end{aligned}
$$

Therefore, we have

$$
K_{2,1}(t) \leq C_{6} t^{-(m+n q+m q) /(2 q)+2(1-b / q)+m / 4} .
$$


For the estimate of $K_{2,2}(t)$, we use $\left(1+|x|^{2}\right)^{p} \geq|x|^{2 p}$ in (3.9). Since

$$
K_{2}(t ; x, y) \leq E\left[e^{-C_{2} t \int_{0}^{1}|x|^{2 p}\left|y+\sqrt{t} Y_{s}\right|^{2 q} d s}\right]
$$

we have

$$
\begin{aligned}
K_{2,2}(t) & \leq t^{2-d / 2} \iint_{|x| \geq 1}\left(1+|x|^{2}\right)^{2 a}|y|^{4 b} E\left[e^{-C_{2} t \int_{0}^{1}|x|^{2 p}\left|y+\sqrt{t} Y_{s}\right|^{2 q} d s}\right]^{1 / 2} d x d y \\
& =t^{2-d / 2} \int_{|x| \geq 1}\left(1+|x|^{2}\right)^{2 a} d x \int|y|^{4 b}(2 \pi t)^{m / 4} J(t ;|x|, y)^{1 / 2} d y
\end{aligned}
$$

where $J(t ;|x|, y)$ is as in Lemma 3.3 (ii) with $c=C_{2}$. If we define

$$
F(t ; x)=(2 \pi t)^{m / 4} \int|y|^{4 b} J(t ;|x|, y)^{1 / 2} d y,
$$

it follows from Lemma 3.3 (ii) with $\lambda=t^{1 / 2}$ and the change of variable $t^{-1 / 2} y \rightarrow y$ that

$$
F(t ; x)=t^{2 b+3 m / 4} F\left(1 ; t^{(1+q) /(2 p)} x\right) .
$$

Thus we have

$$
\begin{aligned}
K_{2,2}(t) & =t^{2-d / 2} \int_{|x| \geq 1}\left(1+|x|^{2}\right)^{2 a} F(t ; x) d x \\
& \leq C_{7} t^{2-d / 2+2 b+m / 2} \int_{|x| \geq 1}|x|^{4 a} F\left(1 ; t^{(1+q) /(2 p)} x\right) d x .
\end{aligned}
$$

A change of variable $t^{(1+q) /(2 p)} x \rightarrow x$ in the last integral leads to

$$
K_{2,2}(t) \leq C_{7} t^{2-d / 2+2 b+m / 2-(1+q)(4 a+n) /(2 p)} \int_{|x| \geq t^{(1+q) /(2 p)}}|x|^{4 a} F(1 ; x) d x .
$$

Here we need the following lemma:

Lemma 3.4. Under the above notations, there exist constants $C_{j}(j=1,2$, 3) such that

$$
F(1 ; x) \leq \begin{cases}C_{1}|x|^{-(4 b+m) p / q}, & \text { for }|x| \leq 1 \\ C_{2} e^{-C_{3}|x|^{2 p /(q+1)}}, & \text { for }|x| \geq 1 .\end{cases}
$$

Proof. Since $J\left(t ;|x|,|x|^{-p /(1+q)} y\right)=|x|^{p m /(1+q)} J\left(|x|^{2 p /(1+q)} t ; 1, y\right)$, we have

$$
\begin{aligned}
F(1 ; x) & =(2 \pi)^{m / 4} \int|y|^{4 b} J(1 ;|x|, y)^{1 / 2} d y \\
& =(2 \pi)^{m / 4} \int|y|^{4 b}|x|^{p m / 2(q+1)} J\left(|x|^{2 p /(q+1)} ; 1,|x|^{p /(q+1)} y\right)^{1 / 2} d y .
\end{aligned}
$$


The change of variable $|x|^{p /(q+1)} y \rightarrow y$ leads to

$$
F(1, x)=(2 \pi)^{m / 4}|x|^{-(4 b+m) p /(q+1)+p m /(2(q+1))} \int|y|^{4 b} J\left(|x|^{2 p /(q+1)} ; 1, y\right)^{1 / 2} d y \text {. }
$$

Since by Lemma 3.3 (i),

$$
\begin{aligned}
& J\left(|x|^{2 p /(q+1)} ; 1, y\right)^{1 / 2} \\
& \leq C_{3}|x|^{-p m / 2(q+1)}\left(e^{-C_{1}|x|^{2 p /(q+1)}|y|^{2 q}}+e^{-C_{2}|y|^{2}|x|^{-2 p /(q+1)}}\right),
\end{aligned}
$$

we have

$$
F(1, x) \leq C_{3}|x|^{-(4 b+m) p /(q+1)} \int|y|^{4 b}\left(e^{-C_{1}|x|^{2 p /(q+1)}|y|^{2 q}}+e^{-C_{2}|y|^{2}|x|^{-2 p /(q+1)}}\right) d y .
$$

By a change of variable $|x|^{p / q(q+1)} y \rightarrow y$ in the first term and $|x|^{-p /(q+1)} y \rightarrow$ $y$ in the second term in the last integral, we have

$$
\begin{aligned}
F(1, x) & \leq C_{4}|x|^{-(4 b+m) p / q} \int|y|^{4 b}\left(e^{-C_{1}|y|^{2 q}}+e^{-C_{2}|y|^{2}}\right) d y \\
& \leq C_{5}\left(|x|^{-(4 b+m) p / q}+1\right) .
\end{aligned}
$$

When $|x| \geq 1$, we write

$$
F(1, x)=(2 \pi)^{m / 4}|x|^{-(8 b+m) p /(2(q+1))} \int|y|^{4 b} J\left(|x|^{2 p /(q+1)} ; 1, y\right)^{1 / 2} d y .
$$

Since $-\frac{1}{2} \Delta_{y}+2 c|y|^{2 q}$ is positive definite, there exists $c_{1}>0$ such that $-\frac{1}{2} \Delta_{y}+2 c|y|^{2 q} \geq 2 c_{1}$. Since $|x| \geq 1$, using Lemma 3.3 (i),

$$
J\left(|x|^{2 p /(q+1)} ; 1, y\right) \leq C_{6} e^{-c_{1}|x|^{2 p /(q+1)}}\left(e^{-C_{3}|y|^{2 q}}+e^{-C_{4}|y|^{2}}\right) .
$$

Therefore, for $|x| \geq 1$, we have

$$
\int|y|^{4 b} J\left(|x|^{2 p /(q+1)} ; 1, y\right)^{1 / 2} d y \leq C_{7} e^{-c_{1}|x|^{2 p /(q+1)}} .
$$

This completes the proof.

End of the proof of Theorem 2.1.

By Lemma 3.4, we have

$$
\begin{aligned}
K_{2,2}(t) \leq & C_{8} t^{2-d / 2+2 b+m / 2-(1+q)(4 a+n) /(2 p)} \int_{|x| \geq t^{(1+q) /(2 p)}}|x|^{4 a} F(1, x) d x \\
\leq & C_{8} t^{2-d / 2+2 b+m / 2-(1+q)(4 a+n) /(2 p)} \\
& \cdot\left\{\int_{t^{(1+q) /(2 p)} \leq|x| \leq 1}|x|^{4 a-(4 b+m) p / q} d x+\int_{|x| \geq 1}|x|^{4 a} e^{-c_{1}|x|^{2 p /(q+1)}} d x\right\} .
\end{aligned}
$$


Therefore, we see that

$$
K_{2,2}(t) \leq\left\{\begin{array}{c}
C_{9} t^{2-d / 2+2 b+m / 2}\left\{t^{-(1+q)(4 a+n) /(2 p)}+t^{-(1+q)(4 b+m) /(2 q)}\right\} \\
\quad \text { if }(4 a+n) q-(4 b+m) p \neq 0 \\
C_{9} t^{2-d / 2+2 b+m / 2-(1+q)(4 a+n) /(2 p)} \log t^{-1} \\
\quad \text { if }(4 a+n) q-(4 b+m) p=0 .
\end{array}\right.
$$

Taking (3.8), (3.10) and (3.13) into consideration, we see that the proof follows.

\section{An Example.}

In this section, we treat the case where the electric potential is of the form (1.3) and the magnetic potential $A(x, y)$ satisfies (A.1) and (A.2).

Let $V(x, y)=\left(1+|x|^{2}\right)^{p}|y|^{2 q}(p, q>0$ integers $)$.

Corollary 4.1. There exists $\delta_{1}>0$ such that:

(i) If $p m>q n, \operatorname{Tr}\left[e^{-t H}\right]=d_{1} t^{-(m+m q+n q) /(2 q)}\left(1+O\left(t^{\delta_{1}}\right)\right)$ as $t \rightarrow 0$.

(ii) If $p m=q n$,

$\operatorname{Tr}\left[e^{-t H}\right]=d_{2} t^{-(m+m q+n q) /(2 q)} \log t^{-1}+d_{3} t^{-(m+m q+n q) /(2 q)}\left(1+O\left(t^{\delta_{1}}\right)\right)$ as $t \rightarrow 0$.

(iii) If $p m<q n, \operatorname{Tr}\left[e^{-t H}\right]=d_{4} t^{-n(1+p+q) /(2 p)}\left(1+O\left(t^{\delta_{1}}\right)\right)$ as $t \rightarrow 0$

where $d_{i}(i=1,2,3,4)$ are given as below.

For the proof, it suffices to note

$$
e^{-t H}=e^{-t H_{0}}+\left(e^{-t H}-e^{-t H_{0}}\right)
$$

and apply Theorem 2.1 and Proposition 1.2.

In order to get the values of the constants $d_{i}$, let $A=-\frac{1}{2} \Delta+|y|^{2 q}$ on $L^{2}\left(\mathbb{R}^{m}\right)$. According to $[\mathbf{3}]$, the complex powers $A^{-s}(s \in \mathbb{C})$ of $A$ are defined for large $\operatorname{Re} s>0$, the trace $\operatorname{Tr}\left[A^{-s}\right]$ has a meromorphic extension $Z_{A}(s)$ in $\mathbb{C}$ whose singularities are all simple poles $\left\{s_{j}=(m(1+q)-j) /(2 q)\right\}_{j=0,1, \ldots}$. Therefore, we can write

$$
Z_{A}(s)=\frac{\operatorname{Res}\left(s_{0}\right)}{s-s_{0}}+C_{(m, q)}+O\left(\left|s-s_{0}\right|\right) \quad \text { as } s \rightarrow s_{0} .
$$


Here $\operatorname{Res}\left(s_{0}\right)$ denotes the residue of $Z_{A}(s)$ at $s_{0}$ and $C_{(m, q)}$ is a constant. Then we have

$$
\begin{aligned}
d_{1}= & 2^{1-d / 2} \frac{1}{q(1+q)} \frac{\Gamma(m /(2 q)) \Gamma((p m-q n) /(2 q))}{\Gamma(m / 2) \Gamma(p m /(2 q))}, \\
d_{2}= & 2^{1-d / 2} \frac{1}{p q} \frac{\Gamma(m /(2 q))}{\Gamma(m / 2) \Gamma(n / 2)}, \\
d_{3}= & 2^{1-d / 2} \frac{1}{\Gamma(n / 2)}\left[\frac{\Gamma(m /(2 q)) \psi((m+m q+n q) /(2 q))}{p q \Gamma(m / 2)}\right. \\
& +C_{(m, q)} \frac{(1+q) \Gamma((m+m q) /(2 q))}{p}-\frac{\gamma \Gamma(m /(2 q))}{q(1+q) \Gamma(m / 2)} \\
& \left.+\frac{\Gamma(m /(2 q))}{p q \Gamma(m / 2)}\left\{\psi\left(\frac{m+m q}{2 q}\right)-\frac{p}{1+q} \psi\left(\frac{n}{2}\right)-\psi\left(\frac{m+m q+n q}{2 q}\right)\right\}\right] \\
d_{4}= & 2^{-n / 2} \frac{(1+q) \Gamma((m+m q) /(2 q)) \Gamma(n(1+p+q) /(2 p))}{p \Gamma(n / 2) \Gamma((m+m q+n q) /(2 q)} Z_{A}\left(\frac{n(1+q)}{2 p}\right)
\end{aligned}
$$

where $\psi(z)=\Gamma^{\prime}(z) / \Gamma(z)$ is the digamma function and $\gamma$ is the Euler constant.

In the particular case where $q=m=1$, we have $s_{0}=1, \operatorname{Res}\left(s_{0}\right)=2^{-1 / 2}$ and $C_{(1,1)}=2^{-1 / 2}(\gamma+\log 2)$. Moreover, for $\operatorname{Re} s>1$, we see that $Z_{A}(s)=$ $\left(2^{s / 2}-2^{-s / 2}\right) R(s)$ where $R(s)$ is the Riemann zeta function. Thus we have

$$
\begin{aligned}
& d_{1}=2^{-(1+n) / 2} \frac{\Gamma((p-n) / 2)}{\Gamma(p / 2)}, \\
& d_{2}=2^{(1-n) / 2} \frac{1}{p \Gamma(n / 2)}, \\
& d_{3}=2^{(1-n) / 2} \frac{1}{\Gamma(n / 2)}\left[\frac{2-p+2^{3 / 2}}{2 p} \gamma-\frac{p}{2} \psi\left(\frac{n}{2}\right)+\frac{2^{1 / 2}}{p} \log 2\right], \\
& d_{4}=2^{1-n / 2} \frac{\Gamma(n(2+p) /(2 p))}{p \Gamma(n / 2) \Gamma(1+n / 2)}\left(2^{n /(2 p)}-2^{-n /(2 p)}\right) R\left(\frac{n}{p}\right) .
\end{aligned}
$$

For a more precise argument, see $[3]$.

\section{References}

[1] J. Aramaki, On an extension of the Ikehara Tauberian theorem, Pacific J. Math., 133(1) (1988), 13-30.

[2] Complex powers of vector valued operators and their applications to asymptotic behavior of eigenvalues, J. Funct. Anal., 87(2) (1989), 294-320.

[3] _ An extension of the Ikehara Tauberian theorem and its application, Acta Math. Hungarica, 71(4) (1996), 297-326.

[4] K. Itô and H.P. Mckean, Jr., Diffusion Processes and Their Sample Paths, Springer, Berlin, Heidelberg, New York, 1974. 
[5] H. Matsumoto, The short time asymptotics of the trace of the heat kernels for the magnetic Schrödinger operators, J. Math. Soc. Japan, 42(4) (1990), 677-689.

[6] _ Classical and non-classical eigenvalue asymptotics for the magnetic Schrödinger operators, J. Funct. Anal., 95 (1991), 460-482.

[7] K. Odencrantz, The effect of a magnetic field on asymptotics of the trace of the heat kernel, J. Funct. Anal., 79 (1988), 398-422.

[8] M. Reed and B. Simon, Method of Modern Mathematical Physics Vol. IV, Analysis of Operators, Academic Press, New York, 1977.

[9] D. Robert, Comportement asymptotique des valeurs propres d'opérateurs du type Schrödinger a potentiel dégénéré, J. Math. Pures et Appl., 61 (1982), 275-300.

[10] M. Schechter, Spectra of Partial Differential Operators, North-Holland, Amsterdam, 1971.

[11] B. Simon, Non-classical eigenvalue asymptotics, J. Funct. Anal., 53 (1983), 84-98.

[12] Semiclassical analysis of low lying eigenvalues II, Tunneling, Ann. of Math., 120 (1984), 89-118.

[13] _ Functional integration and quantum physics, Academic Press, New York, San Francisco, London, 1979.

Received May 17, 1999 and revised September 14, 1999.

Department of Mathematical Sciences

TOKYO DENKI UNIVERSITY

HATOYAMA-MACHI HIKI-GUN

SAITAMA 350-0394

JAPAN

E-mail address: aramaki@r.dendai.ac.jp 